\title{
AVALIAÇÃO DA INFLUÊNCIA DE CARGAS VERTICAIS SOBRE DIFERENTES RODAS COMPACTADORAS NO CICLO DE SEMEADURA DO MILHO
}

\author{
Evaluation of the influence of vertical loads on different press whels \\ in the cycle of sowing of the corn
}

\author{
Jorge Wilson Cortez ${ }^{1}$, Rouverson Pereira da Silva ${ }^{2}$, Alberto Carvalho Filho ${ }^{3}$, \\ Carlos Eduardo Angeli Furlani ${ }^{4}$, Saymon Severino Freitas 5
}

\begin{abstract}
RESUMO
Assim como outras culturas de origem tropical, o milho exige durante o seu ciclo vegetativo, calor e umidade para se desenvolver, e as condições de instalação da cultura podem definir uma boa ou má produção. O objetivo deste trabalho foi avaliar a influência de cargas verticais sobre rodas compactadoras no ciclo da cultura do milho. Este trabalho foi conduzido nas Faculdades Associadas de Uberaba - FAZU, no município de Uberaba (MG), e instalado no delineamento em blocos ao acaso com três modelos de rodas compactadoras e três cargas verticais com duas repetições, na Pista de Ensaios de Semeadura, constituída de canais de solo. Observou-se que o número de dias para emergência, altura de plantas, número de folhas e a produção não foram afetados significativamente para os tratamentos estudados.
\end{abstract}

Termos para indexação: Mecanização, compactação, Zea mays.

\section{ABSTRACT}

As well as other cultures of tropical origin, the corn demands during vegetative cycle, heat and humidity to develop, and the conditions of installation of the culture can define a good or bad yield. The objective of this work was to evaluate the influence of vertical loads on press wheels in the cycle of the culture of the corn. This work was driven at Faculdades Associadas de Uberaba FAZU, in the municipal district of Uberaba (MG), and installed in randomized blocks design with three models of press wheels and three vertical loads with two repetitions, in the Track of Rehearsals of Sowing, constituted of soil channels. Observed that the number of days for emergency, height of plants, number of leaves and the yield were not affected significantly for the studied treatments.

Index terms: Mechanization, compaction, Zea mays.

\section{(Recebido em 22 de novembro de 2005 e aprovado em 20 de dezembro de 2006)}

\section{INTRODUÇÃO}

O milho, uma das poucas culturas econômicas nativas das América, foi encontrado em cultivo pela primeira vez em 05 de novembro de 1492, quando membros da expedição de Colombo, no interior de Cuba retornaram com um tipo de grão amplamente utilizado pelas populações nativas (FORNASIERE FILHO, 1992). Por ser uma planta de origem tropical, o milho, exige durante o seu ciclo vegetativo, calor e umidade para se desenvolver e produzir satisfatoriamente, proporcionando rendimentos compensadores. Os processos da fotossíntese, respiração, transpiração e evaporação, são funções diretas da energia disponível no ambiente, comumente designada por calor; ao passo que o crescimento, desenvolvimento e translocação de fotoassimilados encontram-se ligados à disponibilidade hídrica do solo e seus efeitos são pronunciados em condições de altas temperaturas onde a taxa de evapotranspiração é elevada (FANCELLI \& DOURADO-NETO, 2000).

De acordo com Ritchie et al. (2003), sob condições adequadas no campo, a semente absorve água e começa seu crescimento. A radícula é a primeira a apresentar elongação seguida pelo coleóptilo com a plúmula fechada e as três a quatro raízes seminais laterais. O estádio de emergência é finalmente atingido pela rápida elongação do mesocótilo, o qual empurra o coleóptilo em crescimento para a superfície do solo, sendo que com boas condições de calor e umidade a emergência da plântula ocorrerá dentro de quatro a cinco dias após a semeadura, mas sob condições de temperatura baixas ou de secas, podem ser necessárias duas semanas ou mais.

\footnotetext{
'Engenheiro Agrônomo, Mestre, Doutorando - Departamento de Engenharia Rural - Universidade Estadual Paulista Júlio de Mesquita Filho/UNESP _ Via de Acesso Professor Paulo Donato Castellane, s/n - 14884-900 - Jaboticabal, SP - jorge.cortez@yahoo.com.br

${ }^{2}$ Engenheiro Agrícola, Professor, Doutor- Departamento de Engenharia Rural - Universidade Estadual Paulista Júlio de Mesquita Filho/UNESP - Via de Acesso Professor Paulo Donato Castellane, s/n - 14884-900 - Jaboticabal, SP - rouverson@fcav.unesp.br

${ }^{3}$ Engenheiro Agrônomo, Professor, Doutor - Faculdades Associadas de Uberaba/FAZU - Avenida Tutunas, 720, Tutunas - 38061-500 - Uberaba, MG alberto@fazu.br

${ }^{4}$ Engenheiro Agrônomo, Professor Adjunto, Doutor - Departamento de Engenharia Rural - Universidade Estadual Paulista Júlio de Mesquita Filho/ UNESP - Via de Acesso Professor Paulo Donato Castellane, s/n - 14884-900 - Jaboticabal, SP - furlani@fcav.unesp.br

${ }^{5}$ Graduando em Agronomia - Faculdades Associadas de Uberaba/FAZU - Avenida Tutunas, 720, Tutunas - 38061-500 - Uberaba, MG saymonagro@yahoo.com.br
} 
Para germinação e emergência mais rápidas em semeaduras com datas antecipadas, a semeadura rasa se beneficia das temperaturas do solo mais favoráveis perto da superfície. Em semeaduras mais tardias, as temperaturas do solo são geralmente adequadas para todas as profundidades de semeadura e o teor de água torna-se o fator limitante para o rápido crescimento. Pois as maiores profundidades de semeadura geralmente proporcionam melhor teor de água para as sementes tardias, a menos que tenha ocorrido chuva recentemente (RITCHIE et al., 2003).

Dependendo do teor de água que o solo se encontra o estado da compactação pode-se modificar. Silva (1990) afirma que em solos não compactados a água se encontra retida sob menores tensões, sendo mais susceptíveis ao processo de evaporação na camada superficial, e a condutividade é menor, dificultando a migração de água das camadas inferiores, enquanto que em solos compactados a água é retida sob maior tensão, que retarda a evaporação, a condutividade e facilita a migração de água. Assim, o comportamento hídrico e térmico do solo se altera principalmente em função das cargas, pois as mesmas refletem diferentes estados de compactação.

A compactação do solo agrícola provoca alteração em seu interior modificando o ambiente físico. Salles (1980) relata que a compactação controlada melhora as características de permeabilidade, compressibilidade, condução de água e do calor. Assim, no milho a carga vertical da roda compactadora em torno de $150 \mathrm{~N}$, apresenta-se na cultura como um maior vigor da plântula e maior velocidade de emergência, pois em solos sem pressão ocorre menor taxa de transmissão de água e nutrientes através da interface solo/semente/raiz e o maior índice de velocidade de emergência se deve ao aumento da densidade do solo e seu maior efeito tampão para os nutrientes, ou seja, à medida que baixa a concentração de nutrientes na solução do solo ocorre rápida reposição do nível de nutrientes para a solução do solo, de forma que a planta jovem fica suprida dos nutrientes, evitando assim possível estresse nutricional (SILVA, 1990).

Hummel et al. (1981), em pesquisa com diferentes tipos de rodas compactadoras na semeadura de soja, afirmaram que o desenho da roda apresenta considerável influência, benéfica ou não, sobre o ambiente do solo em torno da semente, e que seu efeito depende do nível de pressão, do desenho, do teor de água, do tipo do solo e das condições climáticas no período entre a semeadura e a emergência.

As rodas compactadoras cilíndricas promovem no solo através da alteração da carga imposta à roda, modificação do condicionamento físico do solo que muda a relação de contato solo-semente (STEFANUTTI et al., 1980).

Cortez et al. (2005) citam que o uso de cargas verticais extremas $(200 \mathrm{~N})$ sobre as rodas compactadoras diminuiu a velocidade e a porcentagem de emergência para a cultura do algodoeiro, e que cargas verticais em torno de 100 a $150 \mathrm{~N}$ trazem benefício ao desenvolvimento inicial das plântulas.

Furlani et al. (2001), combinando três profundidades de semeadura da cultura do milho com quatro níveis de compactação do solo sobre as sementes, não encontraram influência desses fatores sobre o número médio de dias para emergência das plântulas.

O uso de modelos de rodas compactadoras e cargas verticais na operação de semeadura podem influenciar as características físicas do solo alterando a relação de contato solo/semente que poderá modificar o número de dias para emergência, altura de plantas, número de folhas e a produtividade, visto que um estande de plantas mal formadas no início do estabelecimento da cultura acarretará diminuição de produtividade, com este estudo objetivouse avaliar o efeito das cargas verticais sobre as rodas compactadoras, no desenvolvimento da cultura do milho.

\section{MATERIAL E MÉTODOS}

A pesquisa foi conduzida na Pista de Ensaios de Semeadura da FAZU - Faculdades Associadas de Uberaba (MG) cujas coordenadas geodésicas são: Longitude 47²5, W, Latitude $19^{\circ} 45^{\prime} \mathrm{S}$ e altitude de 780 metros. O clima de Uberaba é classificado pelo método de Köeppen como Aw, tropical quente e umido com inverno frio e seco, com médias anuais de temperatura e precipitação de $22^{\circ} \mathrm{C}$ e $1474 \mathrm{~mm}$ respectivamente. A Pista de Ensaios foi desenvolvida por Silva (2002) que possui sete canais de solo planos, separados por trilhos por onde corre um trole com acionamento elétrico com motor de potência de $1,5 \mathrm{cv}$, capaz de realizar as operações de nivelamento, abertura de sulcos para semeadura e compactação sob diferentes níveis de cargas verticais. Os trilhos são espaçados de dois metros entre si com comprimento útil de $24 \mathrm{~m}$.

Cada parcela do experimento foi de 2,2 m com $2 \mathrm{~m}$ de largura totalizando $4,4 \mathrm{~m}^{2}$. Os tratamentos foram montados no Delineamento em Blocos ao acaso no esquema fatorial 3 x 3, com três tipos de rodas e três cargas verticais com duas repetições. As rodas avaliadas (Figura 1) foram: 'V simples' (RD1), chamada de roda dupla angulada constituída por duas rodas cilíndricas em $\mathrm{V}$, revestidas por borracha maciça, normalmente usada para plantio direto; roda ' $\mathrm{V}$ em nervura' 
(RD2) chamada de roda dupla com nervura simples, constituídas por duas rodas cilíndricas, revestidas com borracha maciça e nervura central. Por possuir eixo inclinado essa roda acompanha a forma de um $\mathrm{V}$ e uma 'roda lisa' (RD3) chamada de roda convexa larga, sendo revestida com borracha deformável tipo câmara de ar (SILVA, 1990).

As cargas verticais utilizadas sobre as rodas compactadoras foram a Testemunha (sem carga), 100 e 200 $\mathrm{N}$ que corresponderam as cargas $\mathrm{C} 1, \mathrm{C} 2$ e $\mathrm{C} 3$, respectivamente. Essas cargas foram inseridas nas rodas por meio de massas devidamente mensuradas, adaptadas por um furo central colocando-a sobre seu eixo (Figura 1).

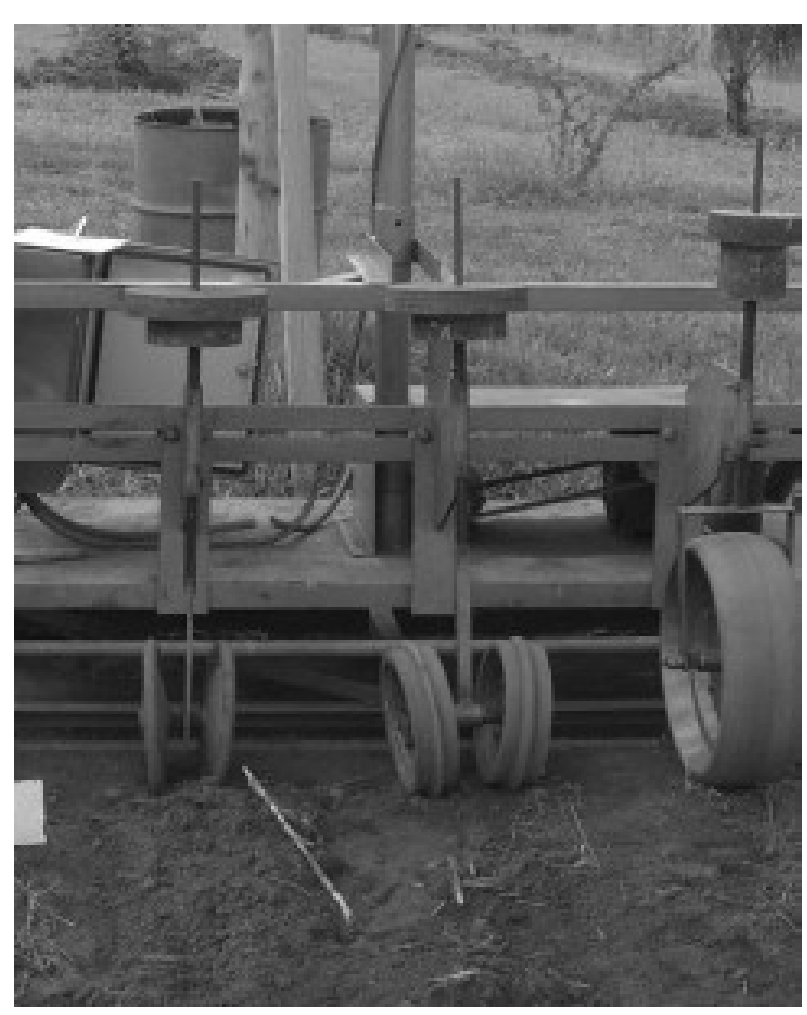

FIGURA 1 - Rodas compactadoras: da esquerda para a direita RD1, RD2 e RD3.

O híbrido de milho utilizado foi da empresa Biomatrix , para produção de grãos, sendo semeado em dezembro de 2004, com espaçamento reduzido de $0,50 \mathrm{~m}$ com seis sementes por metro, proporcionando uma densidade de 120.000 plantas por hectare. Foi feita adubação utilizando a fórmula 2-26-18 na dose de $230 \mathrm{~kg} \cdot \mathrm{ha}^{-1}$. O nitrogênio foi depositado em cobertura na dose de $80 \mathrm{~kg} \cdot \mathrm{ha}^{-1}$. Após a semeadura foram feitas irrigações diárias de uma hora na fase inicial de desenvolvimento, utilizando um aspersor com vazão de $10 \mathrm{~mm} \cdot \mathrm{h}^{-1}$.

Foram coletados dados de emergência das plântulas até o décimo dia após a semeadura, quando ocorreu a estabilização do número de plântulas emergidas. O número médio de dias para emergência foi determinado por meio da equação de Edmond \& Drapala (1958), citados por Siqueira (1999), conforme a Eq. 1.

$$
\mathrm{M}=\frac{[(\mathrm{N} 1 . \mathrm{G} 1)+(\mathrm{N} 2 . \mathrm{G} 2)+\ldots+(\mathrm{Nn} \cdot \mathrm{Gn})]}{(\mathrm{G} 1+\mathrm{G} 2+\cdots+\mathrm{Gn})}
$$

em que:

M - número médio de dias para a emergência das plântulas de milho;

$\mathrm{N}_{1}$ - número de dias decorridos entre a semeadura e a primeira contagem de plântulas;

$\mathrm{G}_{1}$ - número de plântulas emergidas na primeira contagem; $\mathrm{N}_{2}$ - número de dias decorridos entre a semeadura e a segunda contagem de plântulas;

$\mathrm{G}_{2}$ - número de plântulas emergidas entre a primeira e a segunda contagem;

$\mathrm{N}_{\mathrm{n}}$ - número de dias decorridos entre a semeadura e a última contagem de plântulas, e

$\mathrm{G}_{\mathrm{n}}$ - número de plântulas emergidas entre a penúltima e a última contagem.

Foram coletados dados de altura de planta aos 30 dias após emergência tendo a folha bandeira como referência, o número de folhas e a produtividade. Os dados foram submetidos à análise estatística no programa computacional ESTAT desenvolvido pelo Departamento de Ciências Exatas da Faculdade de Ciências Agrárias e Veterinárias de Jaboticabal.

\section{RESULTADOS E DISCUSSÕES}

Os resumos de análise de variância e os resultados médios dos fatores estudados são apresentados em tabelas, nas quais médias seguidas de mesma letra minúsculas não diferem entre si pelo Teste de Tukey a 5 $\%$ de probabilidade.

$\mathrm{Na}$ Tabela 1 são apresentados os valores do número de dias para emergência e observa-se que não houve diferença entre as cargas e os modelos de rodas compactadoras estudadas. Stranak (1968), citado por Justino (1982), afirma que o efeito da compactação na semeadura de cereais até certo nível proporcionou uma germinação mais precoce e uniforme, o que não se observa 
neste experimento. Ao contrário, Carvalho Filho et al. (2005) afirmam que quando as condições de umidade e temperatura são adequadas na fase inicial de desenvolvimento das plântulas não ocorre diferença entre os modelos de rodas.

TABELA 1 - Síntese de médias do número de dias para emergência (NDE).

\begin{tabular}{cc}
\hline Tratamento & NDE \\
\hline Cargas & \\
\hline $\mathrm{C} 1$ & $5,3 \mathrm{a}$ \\
$\mathrm{C} 2$ & $5,4 \mathrm{a}$ \\
$\mathrm{C} 3$ & $5,3 \mathrm{a}$ \\
\hline Rodas & \\
\hline RD1 & $\mathbf{5 , 3} \mathrm{a}$ \\
$\mathrm{RD} 2$ & $5,4 \mathrm{a}$ \\
$\mathrm{RD} 3$ & $\mathbf{5 , 3} \mathrm{a}$ \\
\hline C. V. $(\%)$ & $\mathbf{2 , 6}$ \\
\hline
\end{tabular}

Em cada coluna, para cada fator, médias seguidas de mesmas letras minúsculas não diferem entre si pelo Teste de Tukey a $5 \%$ de probabilidade.

O resultado da análise de emergência pelo teste de F a 5\% não foi significativo para carga (C), rodas (RD) e a interação carga x rodas (C x RD), o que pode ser explicado pelo alto teor de água do solo proporcionado pela irrigação diária e as chuvas de verão. $\mathrm{O}$ efeito da compactação sobre a linha de semeadura é observado quando o solo se encontra em baixas condições de umidade causando selamento e impedindo a emersão da semente.

Silva et al. (2004) afirmam que o número médio de dias para emergência não é afetado pelo modelo de roda compactadora e pela carga vertical devido o experimento ter sido conduzido sob irrigação, o que mascarou a ação destes tratamentos.

Pongelluppi (1979), avaliando a profundidade de semeadura $(0,03,0,04,0,05$ m) e compactação $(87 ; 147 ; 207$ N) na cultura do algodão, observou que não houve diferença estatística na porcentagem de emergência e no NDE para compactação, o que concorda com este experimento.

Dellicer (1984), em seu experimento com a cultura do feijoeiro nas profundidades $(0,02 ; 0,05 ; 0,07 ; 0,1 \mathrm{~m}) \mathrm{e}$ cargas verticais $(0,150,300 \mathrm{~N})$, observou que em relação à emergência a profundidade foi significativa para as camadas de 0,02 e 0,05 m, em relação a camada de 0,01 m.
Para as cargas observou que apenas a de $300 \mathrm{~N}$ diferiu das demais em relação à emergência, contrariando os dados deste experimento.

Segundo Silva (2002), a germinação do milho sofreu diferença estatística apenas na profundidade de semeadura enquanto que rodas e cargas verticais não alteraram o NDE, o que concorda com este experimento.

Na Tabela 2 são apresentados os valores de altura de plantas e número de folhas obtidos para os tratamentos estudados, aos 30 dias após a emergência. Observa-se que não houve efeito para a altura e o número de folhas para as cargas verticais e os modelos de rodas compactadoras, concordando com Rosolem (1995) que afirma, à medida que a planta se aproxima do estádio reprodutivo, se o ambiente for propício, a tendência é de todas as plantas se igualarem, pois a conformação final da planta é determinada geneticamente.

TABELA 2 - Síntese de médias para altura de plantas e número de folhas.

\begin{tabular}{ccc}
\hline Tratamento & Altura (cm) & $\begin{array}{c}\text { Número de } \\
\text { folhas }\end{array}$ \\
\hline Cargas & & \\
\hline C1 & $27,6 \mathrm{a}$ & $6,2 \mathrm{a}$ \\
C2 & $30,2 \mathrm{a}$ & $6,0 \mathrm{a}$ \\
C3 & $29,7 \mathrm{a}$ & $6,1 \mathrm{a}$ \\
\hline Rodas & & \\
\hline RD1 & $27,8 \mathrm{a}$ & $6,1 \mathrm{a}$ \\
RD2 & $29,6 \mathrm{a}$ & $6,1 \mathrm{a}$ \\
RD3 & $30,1 \mathrm{a}$ & $6,1 \mathrm{a}$ \\
\hline C. V. $(\%)$ & $\mathbf{1 0 , 1}$ & 7,7 \\
\hline
\end{tabular}

Em cada coluna, para cada fator, médias seguidas de mesmas letras minúsculas não diferem entre si pelo Teste de Tukey a $5 \%$ de probabilidade.

As rodas não apresentaram diferença estatística os 30 dias, e este resultado concorda com Silva (2002) que não encontrou interferência entre as mesmas rodas em diferentes épocas de avaliação para a cultura do milho. $\mathrm{O}$ número de folhas para a cultura do milho não foi afetado devido às rodas e cargas verticais não interferirem neste processo, pois isso é provavelmente uma característica determinada pela genética da planta.

Nos resultados apresentados não houve diferença entre as os modelos de rodas compactadoras analisadas e 
as cargas verticais impostas na avaliação de produtividade do milho, conforme Tabela 3.

TABELA 3 - Síntese de médias para produtividade da cultura do milho.

\begin{tabular}{cc}
\hline Tratamento & Produtividade $\left(\mathbf{k g . h a}^{\mathbf{- 1}}\right)$ \\
\hline Cargas & $11.700 \mathrm{a}$ \\
$\mathrm{C} 1$ & $8.700 \mathrm{a}$ \\
$\mathrm{C} 2$ & $11.600 \mathrm{a}$ \\
$\mathrm{C} 3$ & \\
\hline Rodas & $10.600 \mathrm{a}$ \\
RD1 & $11.500 \mathrm{a}$ \\
RD2 & $9.900 \mathrm{a}$ \\
RD3 & $\mathbf{2 5 , 5}$
\end{tabular}

Em cada coluna, para cada fator, médias seguidas de mesmas letras minúsculas não diferem entre si pelo Teste de Tukey a $5 \%$ de probabilidade.

Pode-se observar que para as cargas verticais impostas, a produtividade não diferiu mesmo tendo ocorrido um défice hídrico em fevereiro, que provavelmente poderia ter influenciado nas condições de campo fazendo com que cargas maiores proporcionassem melhores níveis de produção. O que não pode ser confirmado já que as cargas $\mathrm{C} 1$ e $\mathrm{C} 3$ obtiveram os melhores resultados correspondendo a testemunha (carga zero) e $200 \mathrm{~N}$, respectivamente.

De modo geral, obteve-se na produção média de 178 sacos por hectare, ou seja, de $10.700 \mathrm{~kg} \cdot \mathrm{ha}^{-1}$, o que demonstra que a cultura do milho pode ser ajustada ao plantio reduzido utilizando população de seis plantas por metro, sem prejudicar a produção, mas sim podendo acarretar aumento na produtividade.

\section{CONCLUSÕES}

O número de dias para emergência não foi alterado pelo modelo da roda compactadora e a carga vertical na hora de semeadura, estando o solo em condições de umidade.

A altura de plantas e o número de folhas não apresentaram diferença entre si, por serem características genéticas.

A produtividade do milho em espaçamento reduzido com seis plantas por metro propiciou produtividade acima da média, mostrando ser uma densidade de plantas satisfatória para a cultura.

\section{REFERÊNCIAS BIBLIOGRÁFICAS}

CARVALHO FILHO, A.; CORTEZ, J. W.; SILVA, R. P.; ZAGO, M. S. Comportamento de cultivares de milho no sistema de plantio direto em diferentes coberturas e rodas compactadoras. Nucleus, Ituverava, v. 3, n. 1, p. 43-45, abr. 2005.

CORTEZ, J. W.; CARVALHO FILHO, A.; SILVA, R. P.; FURLANI, C. E. A. Efeito de cargas verticais exercidas sobre rodas compactadoras na cultura do algodão (Gossypium hirsutum L.). FAZU em Revista, Uberaba, n. 2, p. 45-50, 2005.

DELLICER, C. A. P. Influência da profundidade de semeadura e compactação do solo sobre a semente na emergência, desenvolvimento inicial e produtividade do feijoeiro (Phaseolus vulgaris L.). 1984. 79 f. Monografia (Graduação em Agronomia) - Faculdade de Ciências Agrárias e Veterinárias, Universidade Estadual Paulista, Jaboticabal, 1984.

FANCELLI, A. L.; DOURADO NETO, D. Produção de milho. Guaíba: Agropecuária, 2000. 360 p.

FORNASIERE FILHO, D. A cultura do milho. Jaboticabal: FUNEP, 1992. $273 \mathrm{p}$.

FURLANI, C. E. A.; LOPES, A.; REZENDE, L. C.; SILVA, S. S. S. e; LEITE, M. A. S. Influência da compactação do solo na emergência das plântulas de milho a diferentes profundidades de semeadura. Engenharia na Agricultura, Viçosa, v. 9, n. 3, p. 147-53, 2001.

HUMMEL, J. W.; GRAY, L. E.; NAVE, W. R. Soybean emergence from field seedbed environments. Transactions of the ASAE, Saint Joseph, v. 24, n. 4, p. 872-878, 1981.

JUSTINO, A. Influência da profundidade de semeadura e compactação sobre o solo, na emergência e desenvolvimento inicial do amendoim (Arachis hypogaea L.) variedade TATU. 1982. 91 f. Monografia (Graduação em Agronomia) - Faculdade de Ciências Agrárias e Veterinárias, Universidade Estadual Paulista, Jaboticabal, 1982.

PONGELUPPI, J. B. Influência da profundidade de semeadura e compressão do solo, na emergência e desenvolvimento inicial do algodão (Gossypium hirsutum L.) var. IAC-17. 1979. 53 f. Monografia (Graduação em Agronomia) - Faculdade de Ciências Agrárias e Veterinárias, Universidade Estadual Paulista, Jaboticabal, 1979. 
RITCHIE, S. W.; HANWAY, J. J.; BENSON, G. O. Como a planta de milho se desenvolve. Arquivo do Agrônomo, São Paulo, n. 15, p. 1-20, set. 2003.

ROSOLEM, C. A. Relações solo-planta na cultura do milho. Jaboticabal: Funep, 1995. 53 p.

SALLES, H. C. Influência da compactação do solo no desenvolvimento da soja (Glicine max (l.) Merril). 1980 55 f. Monografia (Graduação a Agronomia) - Faculdade de Ciências Agrárias e Veterinárias, Universidade Estadual Paulista, Jaboticabal, 1980.

SILVA, F. M. Influência do tipo de rodas compactadoras de semeadoras-adubadoras, no condicionamento físico do solo e no desenvolvimento de plantas. 1990. $131 \mathrm{f}$. Dissertação (Mestrado em Engenharia Agrícola) - Faculdade de Engenharia Agrícola, Universidade Estadual de Campinas, Campinas, 1990

SILVA, R. P. Efeito de rodas compactadoras submetidas a cargas verticais em profundidades de semeadura nas características agronômicas do milho (Zea mays L.). 2002.
101 f. Tese (Doutorado em Agronomia) - Faculdades de Ciências Agrária e Veterinárias, Universidade Estadual Paulista, Jaboticabal, 2002

SILVA, R. P.; CORA, J. E.; CARVALHO FILHO, A.; LOPES, A.; FURLANI, C. E. A. Efeito de rodas compactadoras submetidas a cargas verticais em profundidades de semeadura sobre o desenvolvimento do milho. Engenharia na Agricultura, Jaboticabal, v. 24, n. 2, 2004.

SIQUEIRA, R. Sistemas de preparo em diferentes tipos de coberturas vegetais do solo. 1999. $191 \mathrm{f}$. Tese (Doutorado em Agronomia) - Faculdades de Ciências Agronômicas, Universidade Estadual Paulista, Botucatu, 1999.

STEFANUTTI, R.; ORTOLANI, A. F.; COAN, O. Desenvolvimento de uma pista de ensaio e carrinho com rodas compactadoras para estudos relacionados com emergência de plântulas. In: CONGRESSO BRASILEIRO DE ENGENHARIA AGRÍCOLA, 10., 1980, Campinas. Anais... Campinas: FEAGRI, 1980. p. 190203. 